\title{
Dynamic Behavior of Vertical-Cavity Surface-Emitting Lasers
}

\author{
S. F. Yu
}

\begin{abstract}
A quasi-three-dimensional dynamic model of indexguided vertical-cavity surface-emitting lasers is developed. Detailed structure of Bragg reflectors and lateral optical confinement are considered into the model. A three-dimensional waveguide problem is reduced to one dimension by using the effectiveindex method. The dynamic response of optical field is solved by the time-domain algorithm. In addition, the lateral variation of carrier concentration, refractive index, and spontaneous-emission profile are also determined in a self-consistent manner. Using this model, the influence of carrier transport and hot carriers on the dynamic behavior of vertical-cavity surface-emitting lasers is studied. It is found that these nonlinearities have significant influence on the relaxation-oscillation frequency and modulation bandwidth of the devices.
\end{abstract}

\section{INTRODUCTION}

H IGH-MODULATION speed, low-threshold current and single-mode operation are the most attractive features of vertical-cavity surface-emitting lasers (VCSEL's) for the application in optoelectronic integrated circuits. The high-speed performance of VCSEL's is the result of high photon density inside device cavity and hence short stimulated lifetime. It was demonstrated that the relaxation-oscillation frequency (ROF) of VCSEL's can be as high as $71 \mathrm{GHz}$ [1]. However, the maximum modulation bandwidth of VCSEL's is found to be limited to $14 \mathrm{GHz}$ [2]. This low modulation bandwidth of VCSEL's is attributed to the inherent nonlinearities of quantum-well lasers such as carrier diffusion [3], carrier transport [4], and hot carriers [5], as well as self- heating of active layer and Bragg reflectors [6]. In addition, the dynamic response of VCSEL's is also varied with structure and dimension of the laser cavity [2], [7], [8].

Recently, a number of models have been developed, using a simple-rate equations approach with varying degrees of approximation on the optical-field distribution and carrierhole burning [9]-[12], to analyze the transient response of VCSEL's. However, the detailed structure of Bragg reflectors and lateral guidance of the optical-field profile have been ignored in their calculation. In addition, the significant influence of inherent nonlinearities on the modulation response of lasers have not been taken into their analysis. In order to give a guidance for laser design as well as to clarify the influence of device structure and inherent nonlinearities on the dynamic

\footnotetext{
Manuscript received August 3, 1995; revised December 1, 1995. This work was supported by HKU CRCG.

The author is with the Department of Electrical and Electronic Engineering University of Hong Kong, Hong Kong.

Publisher Item Identifier S 0018-9197(96)05035-X.
}

properties of VCSEL's, the development of a detailed model for VCSEL's is required.

In this paper, we analyzed the influence of inherent nonlinearities on the dynamic behavior of VCSEL's. Detailed threedimensional laser structure, including the Bragg reflectors, are considered in our calculation. The three-dimensional problem is converted into one-dimensional traveling-wave equations through the effective-index method [13]. The dependence of lasing conditions on the Bragg reffectors are evaluated through the scattering method, and the dynamic behavior of the traveling waves is solved by the time-domain algorithm. Furthermore, the lateral variation of carrier concentration, refractive index, and spontaneous emission inside the active layer are determined in a self-consistent manner. Using this model, the influence of carrier transport between spacer and active layer as well as injection heating and hot carriers on the modulation response of VCSEL's are analyzed.

This paper is organized as follows: In Section II, we present a self-consistent dynamic model of VCSEL's with the consideration of carrier transport and hot carriers. The lateral variation of carrier concentration, refractive index, and spontaneous-emission profile are taken into calculation. In addition, the longitudinal and lateral distribution of optical field is also modeled. In Section III, the dynamic responses of VCSEL's under the influence of 1) carrier transport and 2) injection heating and hot carriers are compared and analyzed. A brief discussion and conclusion is presented in Section IV.

\section{BASIC EQUATIONS}

\section{A. Device Structure}

The schematic of the VCSEL used in our calculation is shown in Fig. 1. The device has a built-in index-guided structure, and a circular metal contact is formed on the epitaxial side (p-side) for current injection. The GaAs- $\mathrm{AL}_{0.3} \mathrm{Ga}_{0.7} \mathrm{As}$ quantum-well active layer is sandwiched between two undoped spacer layers and two Bragg reflectors, which provide optical feedback for lasing. The undoped spacer layers have thickness of a half-wavelength each, and the Bragg reflectors are formed by alternating layers of AlAs and AlGaAs with quarterwavelength thickness and consist of 15 such pairs on both the $\mathrm{n}$ - and $\mathrm{p}$-side. The active region consists of three GaAs$\mathrm{Al}_{0.3} \mathrm{Ga}_{0.7} \mathrm{As}$ quantum wells with a well width of $100 \AA$ and barrier thickness of $150 \AA$. The corresponding built-in longitudinal and lateral refractive-index distribution of the 


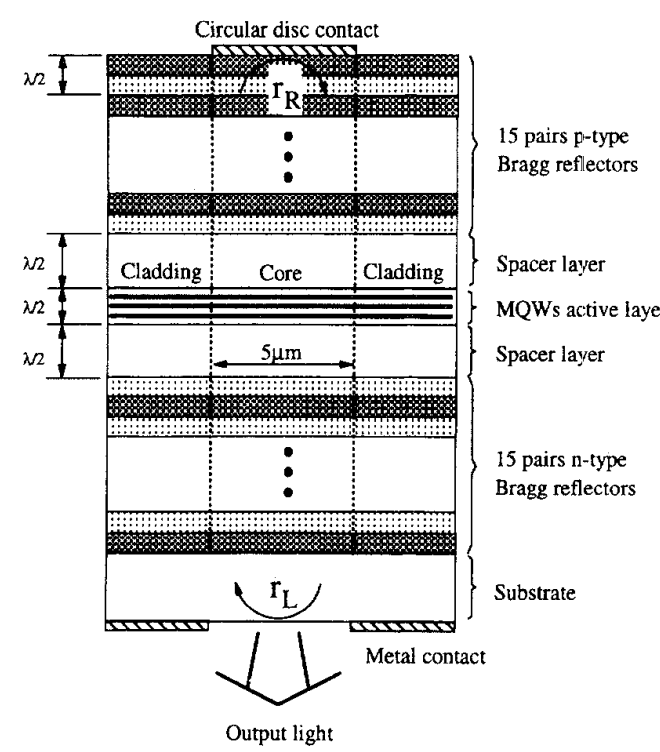

Fig. 1. Schematic of an index-guided VCSEL.

TABLE I

REFRACTIVE-INDEX DistRIBUTION OF VCSEL

\begin{tabular}{c|c|c|c}
\hline Reffactive index of layersiregions & $\begin{array}{c}\text { Core } \\
\text { region }\end{array}$ & $\begin{array}{c}\text { Cladding } \\
\text { region }\end{array}$ & $\begin{array}{c}\text { Effective index } \\
\text { (ne) of each layer }\end{array}$ \\
\hline Active layer & 3.525 & 3.520 & 3.524 \\
\hline Spacer layer & 3.395 & 3.390 & 3.393 \\
\hline High index layer of Bragg reflector & 3.504 & 3.500 & 3.503 \\
\hline Low index layer of Bragg reflector & 2.952 & 2.947 & 2.950 \\
\hline
\end{tabular}

laser is given in Table I. It is assumed that the difference of refractive index between the core and cladding region is around 0.005 for each layer to ensure single lateral-mode operation.

\section{B. Wave Equations}

The space-time evolution of the optical field $E(r, z, t)$ inside the dielectric layers of VCSEL is governed by the wave equation, given as

$$
\left(\frac{\partial^{2}}{\partial z^{2}}+\frac{\partial^{2}}{\partial r^{2}}+\frac{1}{r} \frac{\partial}{\partial r}\right) E=\frac{\varepsilon}{c^{2}} \frac{\partial^{2} E}{\partial t^{2}}+\frac{1}{c^{2}} \frac{\partial \varepsilon}{\partial t} \frac{\partial E}{\partial t}
$$

where $c$ is the velocity of light in free space and $\varepsilon$ is the complex dielectric constant of the laser medium. In (1), it is assumed that the optical field is uniform along the azimuthal direction.

The calculation of the optical field can be further simplified by separating the variable of $r$ and $(z, t)$ such that the corresponding optical field can be expressed as

$$
\begin{aligned}
E(r, z t)= & \Psi(r ; z)\left[A^{+}(z, t) \exp \left(i\left(\omega_{o} t-\beta z\right)\right)\right. \\
& \left.+A^{-}(z, t) \exp \left(i\left(\omega_{o} t+\beta z\right)\right)\right]
\end{aligned}
$$

where $A^{+}(z, t)$ and $A^{-}(z, t)$ are the slowly-varying envelope of the forward- and reverse-traveling waves, respectively, along the longitudinal direction. $\Psi(r ; z)$ is the optical-field distribution along the lateral direction and $\beta$ is the longitudinalpropagation coefficient to be determined. $i$ is a complex number, $\omega_{o}\left(=2 \pi c / \lambda_{o}\right)$ is the lasing frequency, and $\lambda_{o}$ is the operating wavelength. In (2), it is assumed that the lateral field $\Psi(r ; z)$, as well as the propagation coefficient $\beta$, are constant in each index layer. However, all these parameters are varied between layers.

Substitute (2) into (1) and neglect the second derivatives of $A^{ \pm}(z, t)$ with respect to $z$ and $t$, and we get

$$
\begin{aligned}
& {\left[\frac{\partial^{2} \Psi}{\partial r^{2}}+\frac{1}{r} \frac{\partial \Psi}{\partial r}+\left(\varepsilon(r, t ; z) k_{o}^{2}-\beta^{2}\right) \Psi\right] A^{ \pm}} \\
& =2 i \beta\left(\frac{1}{v_{g}} \frac{\partial A^{ \pm}}{\partial t} \pm \frac{\partial A^{ \pm}}{\partial z}\right) \Psi
\end{aligned}
$$

where $k_{o}=\left(2 \pi / \lambda_{o}\right)$ is the wavevector and the group velocity $v_{g}=c / n_{g}$. The group index, $n_{g}$, has an expression of $n_{g}^{2}=\varepsilon+\omega(\partial \varepsilon / \partial \omega) / 2$. Equation (3) can be reduced to one-dimension wave equations in the $z$ direction by the effective-index method [13]. We assumed that the lateral field $\Psi$ satisfies

$$
\begin{aligned}
\frac{\partial^{2} \Psi(r ; z)}{\partial r^{2}}+\frac{1}{r} & \frac{\partial \Psi(r ; z)}{\partial r} \\
& +\left(\varepsilon(r, t ; z) k_{o}^{2}-\beta_{\mathrm{eff}}^{2}(z)\right) \Psi(r ; z)=0
\end{aligned}
$$

where $\beta_{\text {eff }}$ is the effective-propagation coefficient in the lateral direction. By substituting (4) into (3) and integrate over the lateral dimension, we obtain

$$
\frac{1}{v_{g}} \frac{\partial A^{ \pm}}{\partial t} \pm \frac{\partial A^{ \pm}}{\partial z}=-\frac{1}{2} i k_{o} \Delta \varepsilon_{\mathrm{eff}} A^{ \pm}+U_{\mathrm{sp}}^{ \pm}(t) .
$$

In (5), $\Delta \varepsilon_{\text {eff }}$ is defined as

$$
\Delta \varepsilon_{\mathrm{eff}}=\int_{0}^{\infty} \Delta \varepsilon|\Psi(r ; z)|^{2} r d r d \theta / \int_{0}^{\infty}|\Psi(r ; z)|^{2} r d r d \theta
$$

where

$$
\Delta \varepsilon= \begin{cases}\left(-2 \Delta n+i\left(g-\alpha_{s}\right) / k_{o}\right), & \text { active layer } \\ -i \alpha_{s} / k_{o}, & \text { elsewhere }\end{cases}
$$

and $\alpha_{s}$ is the scattering and absorption loss in optical waveguide, $g$ is the optical gain, and $\Delta n$ is the change of refractive index inside the active layer. An extra term, $U_{\mathrm{sp}}^{ \pm}$, is inserted into (5) in order to take into account the spontaneous field coupled to the longitudinal mode, and $U_{\mathrm{sp}}^{ \pm}$only appears within the active layer.

\section{Description of Wave in Bragg Reflectors by Using Scattering Matrix}

The lasing frequency and threshold gain of the device are affected by the reflectivity of Bragg reflectors. The influence of Bragg reflectors on the propagation fields, $A^{+}(z, t)$ and $A^{-}(z, t)$, can be introduced into (5) by the continuity conditions (i.e., matching phase and amplitude of the propagation fields) at the boundary between adjacent dielectric layers. The continuity conditions between layers can be calculated as follows. 




Fig. 2. Reflection and transmission of optical fields between the boundary of the dielectric layers.

1) The equivalent refractive index of each layer is evaluated by the effective-index method [13]. It is noted that the core and cladding regions of each dielectric layer have different refractive-index profiles. The equivalent refractive index, ne, of each layer can be calculated by (4) and is given as

$$
n e=\beta_{\mathrm{eff}} / k_{\circ} .
$$

2) The propagation fields at the boundary between dielectric layers can be determined by the scattering matrix [14]. The longitudinal propagation fields are considered to be reflected or transmitted by the equivalent index discontinuity. It is also assumed that the refractive index is uniform within each layer. At the boundary between two adjacent layers (Fig. 2), the traveling waves can be related by a scattering matrix as

$$
\left[\begin{array}{l}
A_{r}^{+} \\
A_{r}^{-}
\end{array}\right]=\left[\begin{array}{ll}
s_{11} & s_{12} \\
s_{21} & s_{22}
\end{array}\right]\left[\begin{array}{l}
A_{i}^{+} \\
A_{i}^{-}
\end{array}\right]
$$

where $A_{i}^{+}, A_{i}^{-}$and $A_{r}^{+}, A_{r}^{-}$are the incident and reflected waves, respectively. The elements of square matrix in (9) corresponding to the transmission and reflection coefficients at the boundary and these parameters are given as

$$
\begin{aligned}
& s_{11}=s_{22}=2 \sqrt{n e_{l} n e_{h}} /\left(n e_{l}+n e_{h}\right) \\
& s_{21}=-s_{12}=\left(n e_{l}-n e_{h}\right) /\left(n e_{l}+n e_{h}\right)
\end{aligned}
$$

where $n e_{l}$ and $n e_{h}$ are the effective refractive indexes of adjacent layers and can be calculated from (8). In derivation of (10) and (11), only lateral electric field is considered. This is because the fundamental mode of this circular waveguide has no azimuthal variation and $\Psi$ only depends on the lateral position $r$. There is no preferred axis of symmetry in the circular cross section, and the electric field can be directed so that it is everywhere parallel to one of an arbitrary pair of orthogonal directions [15].

\section{Rate Equation of Carrier Concentration Inside the Active and Spacer Layers}

The rate equations for the carrier concentration inside the spacer layer, $N_{1}$, and the active layer, $N_{2}$, are written as [4]

$$
\begin{aligned}
\frac{\partial N_{1}(r, t)}{\partial t}= & \frac{J(r, t)}{e L_{z} N_{w}}-R_{1}(r, t)+\frac{N_{2}(r, t)}{\tau_{21}} \\
& +D_{s} \frac{1}{r} \frac{\partial}{\partial r}\left(r \frac{\partial N_{1}(r, t)}{\partial r}\right) \\
\frac{\partial N_{2}(r, t)}{\partial t}= & \frac{N_{1}(r, t)}{\tau_{12}}-R_{2}(r, t)-v_{g} g\left(N_{2}\right)|E(r, t)|^{2} \\
& +D_{f} \frac{1}{r} \frac{\partial}{\partial r}\left(r \frac{\partial N_{2}(r, t)}{\partial r}\right)
\end{aligned}
$$

where $e$ is the electron charge, $L_{z}$ and $N_{w}$ are the thickness and number of quantum wells, respectively. $\tau_{12}$ is the carrier diffusion and capture time across the spacer layers and $\tau_{21}$ is the thermionic emission time from the quantum well. $D_{f}$ and $D_{s}$ is the ambipolar-diffusion coefficient of the active and spacer layer, respectively. The recombination loss of carrier concentration inside the spacer layers is represented by $R_{1}$ and that in the quantum wells layer is represented by $R_{2}$. The expression of $R_{1}$ and $R_{2}$ are given by

$$
\begin{aligned}
& R_{1}(r, t)=\frac{N_{1}(r, t)}{\tau_{N 1}}+\frac{N_{1}(r, t)}{\tau_{12}} \\
& R_{2}(r, t)=\left[\frac{N_{2}}{\tau_{N 2}}+C_{\text {Aug }} N_{2}^{3}\right]+\frac{N_{2}}{\tau_{21}}+R_{\mathrm{sp}}
\end{aligned}
$$

where $\tau_{N 1}$ and $\tau_{N 2}$ are the carrier lifetime in spacer and active layer, respectively. $R_{\mathrm{sp}}$ is the radiative recombination rate and $C_{\text {aug }}$ is the Auger recombination coefficient.

In $(12), J(r, t)$ is the current-density distribution along the lateral direction. For the case of a circular-disc contact, $J(r, t)$ with the inclusion of current spreading effect can be expressed as [16]

$$
J(r)= \begin{cases}J_{o}(t), & r \leq w \\ J_{o}(t) \exp \left(-(r-w) / r_{o}\right), & r>w\end{cases}
$$

where $J_{0}$ denotes the current density at the edge and within the contact area $(r<w), r_{0}$ is the effective-diffusion length of the injection carrier and $w$ is the radius of circular-disc contact. The value of $r_{0}$ can be determined with the sheet resistance of the entire p-layer Bragg reflectors and the leakage current of the p-n junction.

\section{E. Numerical Technique}

The distribution of $A^{ \pm}(z, t)$ along the laser cavity can be represented by $n+1$ traveling waves (forward and reverse), located at the boundary of dielectric layers, where $n$ is the total number of dielectric layers. If we assume that the thickness of $j$ th dielectric layer is equal to $\Delta z_{j}$ where $j$ is an integer, the position of boundaries between the $j$ th and $j+1$ th layers is $z_{j}+1$, then $z_{1}$ and $z_{n}+1$ are the position of facets. 
The time and spatial variation of wave (5) can be solved by a first-order difference approximation to the partial differential as [17]

$$
\begin{aligned}
\left.\frac{\partial A^{ \pm}}{\partial t}\right|_{z_{j}} \Delta t \pm & \left.\frac{\partial A^{ \pm}}{\partial z}\right|_{t} \Delta z_{j} \\
& \approx A^{ \pm}\left(z_{j} \pm \Delta z_{j}, t+\Delta t\right)-A^{ \pm}\left(z_{j}, t\right)
\end{aligned}
$$

where we have neglected the second derivative $\partial^{2} A^{ \pm} / \partial z \partial t$ in derivation of (17). It is noted that the physical thickness of index layer $\Delta z_{j}$ is varied with its effective refractive index such that their optical length are all equal to quarter wavelength. If we choose the relation between time and spatial steps in the longitudinal direction as $\Delta t=\Delta z_{j} / v_{g j}$ where $v_{g j}$ is the group velocity of $j$ th dielectric layer and $\Delta t$ is a constant time step. Equation (5) can be rewritten as

$$
\begin{aligned}
& A^{ \pm}\left(z_{j} \pm \Delta z_{j}, t+\Delta t\right)-A^{ \pm}\left(z_{j}, t\right) \\
& \quad=\left[-i \Delta \varepsilon_{\mathrm{eff}} k_{o}^{2} A^{ \pm}\left(z_{j}, t\right) / 2 \beta+U_{\mathrm{sp}}^{ \pm}(t)\right] \Delta z_{j} .
\end{aligned}
$$

Now, the longitudinal distribution of traveling waves can be calculated by (18) and (9).

In the program, the traveling waves, $A^{ \pm}$, are advanced from one dielectric interface to another [using (18)] at each time interval, $\Delta t$. At the same instant, the boundary conditions are applied to the propagation fields at the dielectric interface [by (9)] for the calculation of the reflectivity of the alternating dielectric layers. Therefore, the reflectivity of Bragg reflectors is evaluated in a time-dependent manner. For the output power, the boundary conditions at the top and bottom surface of the Bragg reflectors can be written as

$$
\begin{aligned}
A^{-}\left(t, z_{n+1}\right) & =r_{R} A^{+}\left(t, z_{n+1}\right) \quad \text { and } \\
A^{+}\left(t, z_{1}\right) & =r_{L} A^{-}\left(t, z_{1}\right)
\end{aligned}
$$

where $r_{R}$ and $r_{L}$ is the reflectivity between semiconductormetal and air-semiconductor interface (Fig. 1), respectively.

The carrier distribution $N_{1,2}(r, t)$ along the lateral direction of the active layer can be represented from $r_{1}=0$ to $r_{m}=$ $(m-1) \Delta r$ by $m$ elements, where $\Delta r$ is the separation between two successive points in the $r$ direction. The derivative of carrier concentration at $j$ th element (i.e., at $r=r_{j}$ ) can be also approximated by the finite difference

$$
\begin{aligned}
\left.\frac{1}{r_{j}} \frac{\partial N_{1,2}}{\partial r}\right|_{r_{m}} & =\frac{N_{1,2}\left(r_{j+1}, t\right)-N_{1,2}\left(r_{j}, t\right)}{(j-1) \Delta r^{2}} \\
\left.\frac{\partial^{2} N_{1,2}}{\partial r^{2}}\right|_{r_{j}} & =\frac{N_{1,2}\left(r_{j+1}, t\right)-2 N_{1,2}\left(r_{j}, t\right)+N_{1,2}\left(r_{j-1}, t\right)}{\Delta r^{2}}
\end{aligned}
$$

for $j>1$. The time variation of carrier concentration at $j$ th element can also be approximated by

$$
\left.\frac{\partial N_{1,2}}{\partial t}\right|_{r_{j}} \Delta t=N_{1,2}\left(r_{j}, t+\Delta t\right)-N_{1,2}\left(r_{j}, t\right)
$$

where $\Delta t$ is identical to that given in (17) and the time variation of carrier concentration is synchronized with the traveling waves. By substituting (20), (21), and (22) into (12), the rate of carrier concentration $N_{1}$ can be written as

$$
\begin{aligned}
N_{1}\left(r_{j}, t\right. & +\Delta t)-N_{1}\left(r_{j} t\right) \\
= & \Delta t\left\{\frac{J\left(r_{j}, t\right)}{e L_{z} N_{w}}-R_{1}\left(r_{j}, t\right)+\frac{N_{2}\left(r_{j}, t\right)}{\tau_{21}}\right. \\
& +\frac{D_{s}}{\Delta r^{2}}\left[\frac{\left(N_{1}\left(r_{j+1}, t\right)-N_{1}\left(r_{j}, t\right)\right)}{j-1}\right. \\
& \left.\left.+\left(N_{1}\left(r_{j+1}, t\right)-2 N_{1}\left(r_{j}, t\right)+N_{1}\left(r_{j-1}, t\right)\right)\right]\right\}
\end{aligned}
$$

and (13) can also be expressed in a similar format.

The time dependent carrier-rate equations are solved subject to the conditions that $N_{1,2}$ and its derivative are continuous everywhere and at the element $j=1$, the first and second derivative take the form

$$
\begin{aligned}
\left.\frac{\partial N_{1,2}}{\partial r}\right|_{r_{1}} & =0 \\
\left.\frac{\partial^{2} N_{1,2}}{\partial r^{2}}\right|_{r_{1}} & =\frac{2\left(N_{1,2}\left(r_{2}, t\right)-N_{1,2}\left(r_{1}, t\right)\right)}{\Delta r^{2}} .
\end{aligned}
$$

This is because of the circular symmetry of the carrier concentration. It is also required that as the total element number $m \rightarrow$ very large, the carrier concentration change $\Delta N_{1,2} \rightarrow 0$.

\section{F. Gain Spectrum, Induced Refractive-Index Change, and Spontaneous-Emission Rate}

The effect of valence band mixing on the subband wavefunctions and dispersion can be calculated by $k \cdot p$ method. Using the density-matrix approach, the optical gain with photon generated in the direction perpendicular to the surface of quantum-well layers is given as

$$
\begin{aligned}
g(\omega)= & \frac{e^{2} M_{b}^{2}}{\pi c \varepsilon m_{o}^{2} \omega L_{z}} \sum_{p, q} \int\left|\left\langle\psi_{C p} \mid \psi_{V q}\right\rangle\right|^{2} \mathcal{P}_{p q}(k) \\
& \times \mathcal{L}\left[E_{p}(k)-E_{q}(k)-\hbar \omega\right] \\
& \times\left\{f^{c}\left[E_{p}(k)\right]-f^{v}\left[E_{q}(k)\right]\right\} d k
\end{aligned}
$$

where $m_{o}$ is the rest mass of electron and $M_{b}$ is the optical matrix. $E_{p}$ and $E_{q}$ are the $p$ th-electron and $q$ th-hole subbandedge energy, respectively, and $\psi_{c}$ and $\psi_{v}$ are the envelope wavefunctions for the electrons and holes, respectively. $\mathcal{L}$ is the Lorentzian broadening factor with HWHM of $5 \mathrm{meV}$ and $k$ is the wavevector. The summation in (26) is over all the conduction and valence subbands, and $\mathcal{P}(k)$ is the unpolarization factor. It must be noted that the value of $\mathcal{P}(k)$ is the same as the TE-polarization factor due to the orientation of the circular waveguide, as the lateral field is always parallel to the plane of quantum wells. $f^{c}$ and $f^{v}$ are the quasiFermi for the electrons in the conduction and valance bands, respectively. The bandgap shrinkage caused by the injected carriers is also taken into account using an $N^{1 / 3}$ dependence. The method of gain-spectrum calculation is similar to that in [18]. Fig. 3 shows the optical-gain spectra for various levels of injection-carrier concentration. The temperature of 


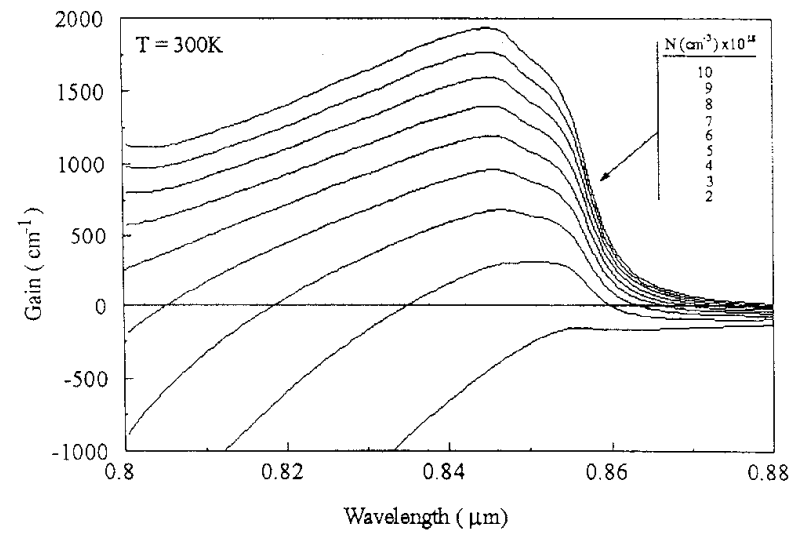

Fig. 3. Calculated optical-gain spectrum of $\mathrm{GaAs}-\mathrm{Al}_{0.3} \mathrm{Ga}_{0.7}$ As quantum well at room temperature with various levels of injection-carrier concentration.

the quantum well is assumed to be constant and is equal to $300 \mathrm{~K}$ through the whole calculation process.

The optical gain given in (26) can be implemented into (7) with the following procedures:

1) The dependence of peak gain on injection-carrier concentration can be approximated by

$$
g=a_{N} \log \left(N_{2} / N_{o}\right)
$$

where $a_{N}$ is a fitted parameter and $N_{o}$ is the carrier concentration at transparency. These parameters can be determined from Fig. 3. It is found that $a_{N}$ and $N_{0}$ are equal to $1309.8 \mathrm{~cm}^{-1}$ and $2.3572 \times 10^{18} \mathrm{~cm}^{-3}$, respectively.

2) The frequency-dependent gain can be modeled using a digital filter. A possible digital filter with unit peak gain is [19]

$$
y_{t+\Delta t}=B y_{t}+(1-B) x_{t}
$$

where $y_{t}$ and $x_{t}$ are the output and input of the digital filter at time $t$, respectively. $B$ is a complex number with $|B|<1$. The phase of $B$ determines the peakgain frequency, and the magnitude of $B$ determines the bandwidth of the digital filter. We assume $B=$ $b \exp \left(i \omega_{p} \Delta t\right)$, where $b$ is a fitted parameter and $\omega_{p}$ is the peak frequency. The frequency response of this filter is

$$
g(\omega)=\frac{(1-b)^{2}}{\left.1+b^{2}-2 b \cos \left(\omega-\omega_{p}\right) \Delta t\right)} .
$$

The parameter $B$ can be estimated by numerically fitting the calculated gain curve as shown in Fig. 3 with this digital-filter gain. The peak frequency, $\omega_{p}$ is also a function of injection-carrier concentration and can be approximated by

$$
\omega_{p}=\omega_{p 0}+\Delta \omega_{p}
$$

where the constant $\omega_{p 0}$ and $\Delta \omega_{p}$ can be determined from Fig. 3.
The change of refractive index $\Delta n(\omega)=n(\omega)-n_{o}(\omega)$ can be calculated from the change of gain coefficient $\Delta g(\omega)=$ $g(\omega)-g_{0}(\omega)$ through the Kramers-Kronig dispersion relation [20]

$$
\Delta n(\omega)=\frac{\pi}{c} P V \int_{0}^{\infty} \frac{\Delta g\left(\omega^{\prime}\right)}{\omega^{\prime 2}-\omega^{2}}
$$

where $n_{o}(\omega)$ and $g_{o}(\omega)$ are the refractive index and optical gain at transparency, respectively. The symbol $P V$ stands for the Cauchy principle value.

Gain suppression can also be introduced into our model by assuming that it is independent of the carrier concentration, and the optical gain can be written by

$$
g=\frac{a_{N} \log \left(N_{2} / N_{o}\right)}{1+\varepsilon_{s}|E|^{2}}
$$

where $\varepsilon_{s}$ is the gain suppression factor.

The spontaneous-emission rate (of unit $\mathrm{cm}^{-3} \mathrm{~s}^{-1}$ ) generated inside the quantum-well layers is given by

$$
\begin{aligned}
\mathcal{R}_{\mathrm{sp}}= & \int d(\hbar \omega) \frac{\varepsilon \omega e^{2} M_{b}^{2}}{2 \hbar \pi^{3} c^{2} m_{o}^{2}} \sum \int\left|\left\langle\psi_{C p} \mid \psi_{V q}\right\rangle\right|^{2} \mathcal{P}_{p q}(k) \\
& \times \mathcal{L}\left[E_{p}(k)-E_{q}(k)-\hbar \omega\right] \\
& \times\left\{f^{c}\left[E_{p}(k)\right]\right\}\left\{1-f^{v}\left[E_{q}(k)\right]\right\} d k
\end{aligned}
$$

and the spontaneous fields, $U_{\mathrm{sp}}^{ \pm}(t)$, coupled into the longitudinal mode can be estimated with the following assumptions [17].

1) The spontaneous-emission fields coupled to the forward and reverse waves have equal amplitude (i.e., $U_{\mathrm{sp}}(t)=$ $\left.U_{\mathrm{sp}}^{+}(t)=U_{\mathrm{sp}}^{-}(t)\right)$

2) The emissions have a Gaussian distribution and satisfy the correlation

$$
\begin{aligned}
& \left\langle U_{\mathrm{sp}}(t) U_{\mathrm{sp}}^{*}\left(t^{\prime}\right)\right\rangle=C_{s} R_{\mathrm{sp}} \delta\left(t-t^{\prime}\right) / v_{g} / L_{z} N_{w} \\
& \left\langle U_{\mathrm{sp}}(t) U_{\mathrm{sp}}\left(t^{\prime}\right)\right\rangle=0
\end{aligned}
$$

where $\delta(t)$ is defined as the delta function and $C_{s}$ is the spontaneous-emission factor of the longitudinal mode. $R_{\mathrm{sp}}$ can be calculated from (34) through the relation

$$
R_{\mathrm{sp}}=\int_{0}^{\infty} \mathcal{R}_{\mathrm{sp}}|\Psi(r)|^{2} r d r / \int_{0}^{\infty}|\Psi(r)|^{2} r d r .
$$

In (34), the spontaneous emission is only coupled to the traveling waves inside the quantum wells. The enhancement of spontaneous emission inside the microcavity can be taken into calculation with appropriate estimation of $C_{s}[21]$.

\section{G. Injection Heating and Hot Carriers}

In quantum-well VCSEL's, the main source of external heating is caused by the injection of energetic carriers into the active region. This is because of small cavity dimensions and high injection-current density within the quantum well. Relaxation of these energetic carriers via intercarrier collisions and optical-phonon emission heating both carriers and phonons. The dynamic behavior of lasers as well as optical gain are varied with the temperature of carriers and phonons. Especially 
at high density of carrier injection, the role of hot carriers cannot be neglected [5]. In this section, we investigate the influence of injection heating and hot carriers on the dynamic performance of VCSEL's.

We adopt the analytical model given in [5], and the dynamic behavior of VCSEL's is investigated with the consideration of injection heating and hot-carrier effects. It is assumed that the populations of carriers, phonons, and photons are dynamically coupled together. Therefore, the dynamic behavior of carriers, phonons, and photons can be calculated by the corresponding rate equations.

The equation describing energy relaxation is given by

$$
\begin{gathered}
\frac{N}{N_{r}} \frac{d N}{d t}+\frac{\pi^{2}}{3} k_{B}^{2} T_{e}\left(N_{e}+N_{h}\right) \frac{d T_{e}}{d t} \\
=-\frac{\hbar \omega_{\mathrm{LO}}}{\tau_{s}}\left[n_{e}\left(\omega_{\mathrm{LO}}\right)-n\left(\omega_{\mathrm{LO}}\right)\right] \\
\cdot\left(N_{e}+N_{h}\right) \hbar \omega_{\mathrm{LO}}+F J
\end{gathered}
$$

where $N_{e}, N_{h}$ are the density of states per unit energy interval in the conduction and valence subbands and can expressed as [22]

$$
N_{e / h}=\frac{m_{c / h}^{*}}{\pi \hbar^{2} L_{z}} \approx 4.168 \times 10^{20} \frac{m_{e / h}^{*}}{m_{o}}
$$

where $m_{e / h}^{*}$ is the effective mass of electron/hole and $L_{z}(=$ $100 \AA$ ) is the width of the quantum well. $N_{r}$ is the reduced density of states per unit energy interval, $k_{B}$ is the Boltzmann constant, and $T_{e}$ is the temperature of the electron. $\omega_{\mathrm{LO}}$ is the average phonon frequency of LO modes and $\tau_{s}$ is the phononscattering time constant. $n\left(\omega_{\mathrm{LO}}\right)$ is the occupation number of phonon and $n_{e}\left(\omega_{\mathrm{LO}}\right)$ is the Bose-Einstein occupation number when the phonon temperature equals the electron temperature and is given by

$$
n_{e}\left(\omega_{\mathrm{LO}}\right)=\frac{1}{\exp \left(\hbar \omega_{\mathrm{LO}} / k_{B} T_{e}\right)-1}
$$

The effective electric field $F=\Delta E_{g} / e d$ and $\Delta E_{g}$ is the bandgap discontinuities in the well minus the subband energies and this parameter $\left(\Delta E_{g}\right)$ describes the effects of junction heating.

The phonon-occupation rate is described by

$$
\begin{aligned}
\frac{d n\left(\omega_{\mathrm{LO}}\right)}{d t}= & \Gamma_{p n} \frac{\hbar \omega_{\mathrm{LO}}}{\tau_{s}}\left[n_{e}\left(\omega_{\mathrm{LO}}\right)-n\left(\omega_{\mathrm{LO}}\right)\right] \\
& \cdot \frac{\left(N_{e}+N_{h}\right)}{2 N}-\frac{\left[n\left(\omega_{\mathrm{LO}}\right)-n_{o}\left(\omega_{\mathrm{LO}}\right)\right]}{\tau_{p n}}
\end{aligned}
$$

where $\Gamma_{p n}$ is the confinement factor of phonon modes inside the active region, and $n_{o}\left(\omega_{\mathrm{LO}}\right)$ is the occupation number at thermodynamic equilibrium and can be calculated from

\begin{tabular}{|c|c|c|}
\hline Parameter $^{+}$ & Symbol & Formulas \\
\hline effective mass (GaAs) of electrou & $\mathrm{m}_{\mathrm{e}(\mathrm{GaAs})}^{*}$ & $0.0665-1.848 \times 10^{-5} \times \mathrm{T}^{2} /(\mathrm{T}+204)$ \\
\hline effective mass (GaAs) of light hole & $\mathrm{m}_{1 \mathrm{~h}(\mathrm{GaAs})}^{*}$ & $0.0951-3.902 \times 10^{-5} \times \mathrm{T}^{2} /(\mathrm{T}+204)$ \\
\hline effective mass (GaAs) of heavy hole & $\mathrm{m}_{\mathrm{hh}(\mathrm{GaAs})}^{*}$ & $\begin{array}{l}0.3319624+0.002029 \times \mathrm{T} \\
-4.896 \times 10^{-6} \times \mathrm{T}^{2} \\
\end{array}$ \\
\hline effective mass $\left(\mathrm{Al}_{\mathrm{x}} \mathrm{Ga}_{1-\mathrm{x}} \mathrm{As}\right)$ of electron & $\mathrm{m}_{\mathrm{e}(\mathrm{AlGaAs})}^{*}$ & 0.09096 \\
\hline effective mass $\left(\mathrm{Al}_{\mathrm{x}} \mathrm{Ga}_{1-\mathrm{x}} \mathrm{As}\right)$ of light hole & $\mathrm{m}_{\mathrm{la}(\mathrm{AlGaAs})}^{*}$ & 0.08802 \\
\hline effective mass $\left(\mathrm{Al}_{\mathrm{x}} \mathrm{Ga}_{1-\mathrm{x}} \mathrm{As}\right)$ of heavy hole & $\mathrm{m}_{\mathrm{hh}(\mathrm{AlG} \mathrm{GAs})}^{*}$ & $0.56+6.803 \times 10^{-4} \times \mathrm{T}$ \\
\hline energy bandgap of GaAs & $E_{g(G a A s)}$ & $1.519-5.408 \times 10^{-4} \times \mathrm{T}^{2} /(\mathrm{T}+204)$ \\
\hline energy bandgap of $\mathrm{Al}_{\mathrm{x}} \mathrm{Ga}_{1-\mathrm{x}} \mathrm{As}$ & $E_{g(A \backslash G a A s)}$ & 1.884634 \\
\hline permittivity of GaAs & $\varepsilon_{\text {of(GaAs })}$ & $12.40 \times\left(1+1.2 \times 10^{-4} \times \mathrm{T}\right)$ \\
\hline permittivity of $\mathrm{Al}_{\mathrm{x}} \mathrm{Ga}_{1-\mathrm{x}} \mathrm{As}$ & $\varepsilon_{O(A \cup G a A S)}$ & 12.24 \\
\hline
\end{tabular}
(38) with $T_{e}$ replaced by the lattice temperature. $\tau_{p n}$ is the corresponding phonon lifetime. Equations (36) and (39) describe the influence of recombination heating and hotphonon effects on the dynamic behavior of lasers.
TABLE II

PARAMETERs OF THE GaAs-AlGaAs QW Material

TABLE III Fitted Parameters of the Optical Gain

\begin{tabular}{c|c|c|c}
\hline Symbol & Value & Symbol & Value \\
\hline $\mathrm{a}_{0}$ & $2478.3 \mathrm{~cm}^{-1}$ & $\mathrm{c}_{0}$ & $-2.2199 \times 10^{-2}$ \\
\hline $\mathrm{a}_{1}$ & $-7.6369 \mathrm{~cm}^{-1} \mathrm{~K}^{-1}$ & $\mathrm{c}_{1}$ & $-4.6154 \times 10^{-6} \mathrm{~K}^{-1}$ \\
\hline $\mathrm{a}_{2}$ & $1.3177 \times 10^{-2} \mathrm{~cm}^{-1} \mathrm{~K}^{-2}$ & $\mathrm{c}_{2}$ & $-3.0319 \times 10^{-9} \mathrm{~K}^{-2}$ \\
\hline $\mathrm{b}_{0}$ & $8.4226 \times 10^{17} \mathrm{~cm}^{-3}$ & $\mathrm{~d}_{0}$ & $1.2059 \times 10^{18} \mathrm{~cm}^{-3}$ \\
\hline $\mathrm{b}_{1}$ & $5.0679 \times 10^{15} \mathrm{~cm}^{-3} \mathrm{~K}^{-1}$ & $\mathrm{~d}_{1}$ & $4.3653 \times 10^{16} \mathrm{~cm}^{-3} \mathrm{~K}^{-1}$ \\
\hline
\end{tabular}

A complete set of rate equations can be obtained with the addition of traveling waves and carrier-rate equations. The dynamic behavior of VCSEL's under the influence of injection heating and hot carriers can be calculated by solving these rate equations self-consistently. The optical gain used in the traveling waves and carrier-rate equations is now also a function of electron temperature.

The temperature dependence of optical gain can be calculated from (26), however, the knowledge of temperature dependence of electron/hole mass, Fermi energy level, as well as bandgap have to be determined before the computation of optical gain. A list of these empirical formulas are given in Table II, and the temperature dependence of optical gain can be estimated.

Using the above parameters, a simple expression of optical gain with the dependent of temperature can be obtained (by curve fitting) and is given by

$$
g=a_{N} \log \left(N_{2} / N_{o}\right)
$$

and the temperature dependence of $a_{N}$ and $N_{o}$ are expressed as

$$
\begin{aligned}
& a_{N}(T)=a_{0}+a_{1} T+a_{2} T^{2} \\
& N_{o}(T)=b_{0}+b_{1} T
\end{aligned}
$$

where $a_{o}, a_{1}, a_{2}, b_{o}$ and $b_{1}$ are some constants and can be found in Table III. In addition, the change of refractive index, $\Delta n$, with the variation of temperature can also be deduced 
TABLE IV

LASER Parameters

\begin{tabular}{|c|c|c|}
\hline Parameter & Symbol & Value \\
\hline radius of the circular contact & $w$ & $2.5 \mu \mathrm{m}$ \\
\hline effective diffusion length of injection carrier & $\mathrm{r}_{0}$ & $0.02 \mu \mathrm{m}$ \\
\hline lasing wavelength & $\lambda_{0}$ & $0.85 \mu \mathrm{m}$ \\
\hline velocity of light in free space & c & $3 \times 10^{10} \mathrm{~cm} / \mathrm{s}$ \\
\hline scattering \& absorption loss in waveguide & $\alpha_{s}$ & $10 \mathrm{~cm}^{-1}$ \\
\hline spontaneous emission factor & $\mathrm{C}_{\mathrm{s}}$ & 0.001 \\
\hline gain compression factor & $\varepsilon_{s}$ & $0.5 \times 10^{-17} \mathrm{~cm}^{3}$ \\
\hline carrier lifetime in active layer & $\tau_{\mathrm{N} 1}$ & $3 \times 10^{-9} \mathrm{~s}$ \\
\hline carrier lifetime in spacer layer & $\tau_{\mathrm{N} 2}$ & $3 \times 10^{-9} \mathrm{~s}$ \\
\hline Auger recombination rate & $\mathrm{C}_{\text {aug }}$ & $3 \times 10^{-28} \mathrm{~cm}^{6} \mathrm{~s}^{-1}$ \\
\hline phonon scattering time constant ${ }^{+}$ & $\tau_{s}$ & $170 \mathrm{fs}$ \\
\hline phonon lifetime ${ }^{+}$ & $\tau_{\mathrm{pm}}$ & $4 \mathrm{ps}$ \\
\hline average phonon energy of LO modes ${ }^{+}$ & $\hbar \omega_{20}$ & $34 \mathrm{meV}$ \\
\hline phonou confinement factor ${ }^{+}$ & $\Gamma_{p n}$ & 0.158 \\
\hline lef facet reflectivity & $r_{L}$ & 0.55 \\
\hline right facet reflectivity & $I_{R}$ & 0.974 \\
\hline
\end{tabular}

from the Kramers-Kronig dispersion relation [20]. A simple expression of $\Delta n$ is given by

$$
\Delta n=b_{N}(T) \log \left(N_{2} / N_{b}(T)\right)
$$

and the temperature dependence of $a_{N}$ and $N_{b}$ are given as

$$
\begin{aligned}
& b_{N}(T)=c_{0}+c_{1} T+c_{2} T^{2} \\
& N_{b}(T)=d_{0}+d_{1} T
\end{aligned}
$$

where $c_{0}, c_{1}, c_{2}, d_{o}$, and $d_{1}$ are some constants and can also be found in Table III. The peak-wavelength shift due to the variation of temperature of the active region is also approximated by

$$
\Delta \omega_{p}=-\frac{2 \pi c}{\lambda_{p}^{2}}\left(\frac{\partial \lambda}{\partial T} \Delta T+\frac{\partial \lambda}{\partial N_{2}} \Delta N_{2}\right)
$$

where $\lambda_{p}$ is the peak wavelength and $\Delta T$ and $\Delta N_{2}$ are the small change of temperature and carrier concentration above threshold. The magnitude of $\lambda_{p}, \partial \lambda / \partial T$ and $\partial \lambda / \partial N_{2}$ can also be estimated from the temperature-dependent gain curve (by curve fitting).

\section{NUMERICAL RESUltS}

In the following analysis, we assumed that the injection current is well confined in the core region and $r_{o}$ given in (16) is set to $0.02 \mu \mathrm{m}$. It can be shown that the modulation response of the lasers is less dependent on $r_{0}$. Other device parameters used in the following calculations can be found in Table IV.

\section{A. The Large-Signal Dynamic Behavior of VCSEL's}

A typical large-signal dynamic response of VCSEL's is shown in Fig. 4. In our calculation, the influence of carrier transport and hot carriers have been ignored. It is assumed that the laser is initially biased at threshold and modulated with a step-current. Fig. 4(a) shows the transient response of optical power with damped oscillations. The frequency



(a)

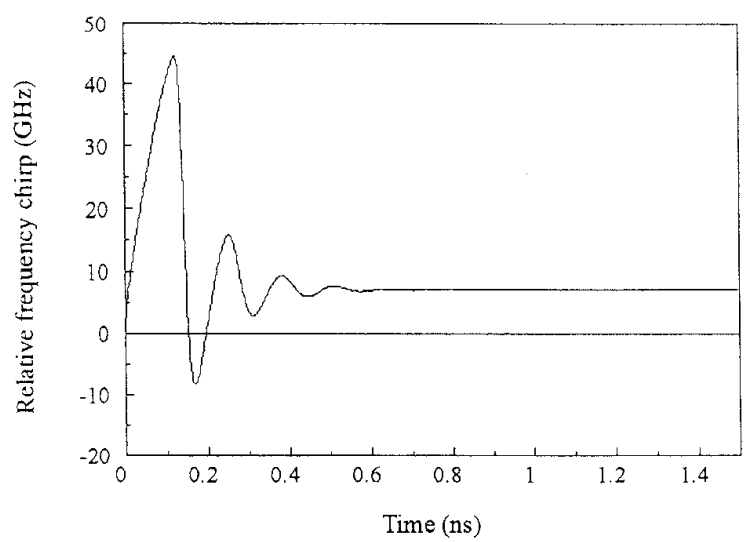

(b)

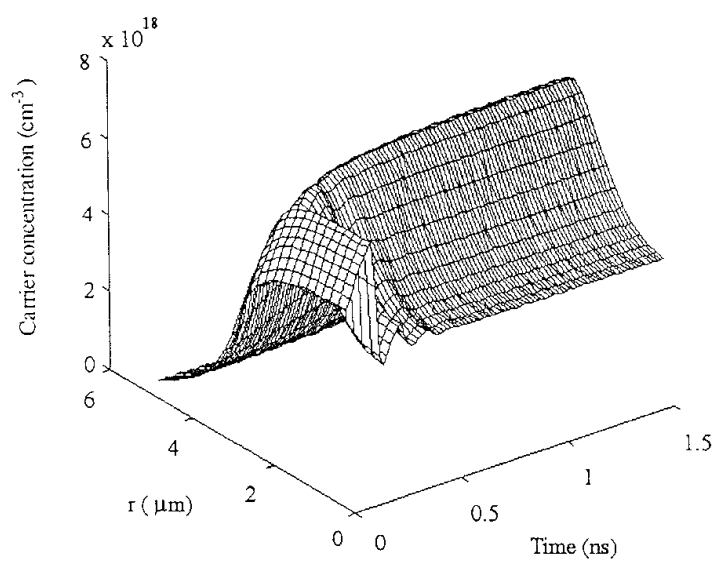

(c)

Fig. 4. Time evolution of (a) output power, (b) transient chirp, and (c) lateral carrier concentration of VCSEL with $D_{f}=5 \mathrm{~s}^{-1} \mathrm{~cm}^{2}$.

chirp during the laser switch-on is given in Fig. 4(b), and the frequency change is caused by the variations of refractive index inside the active layer that is related to changes of the carrier concentration, as given by (42). The time evolution of lateral variation of carrier concentration is also shown in Fig. 4(c). Lateral spatial-hole burning (LSHB) of carrier density occurrs during the first overshoot of optical power due to the stimulated recombination. 


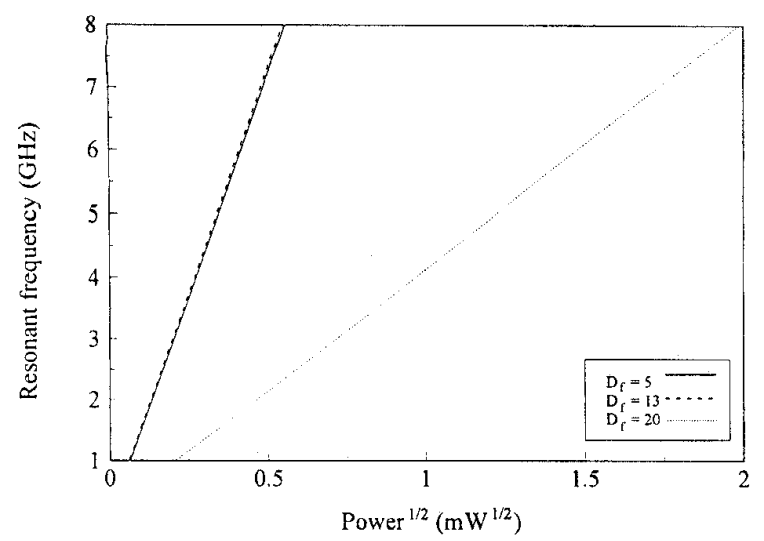

Fig. 5. Relaxation-oscillation frequency of VCSEL's (without carrier transport) as a function of squared root of the steady-state optical power.

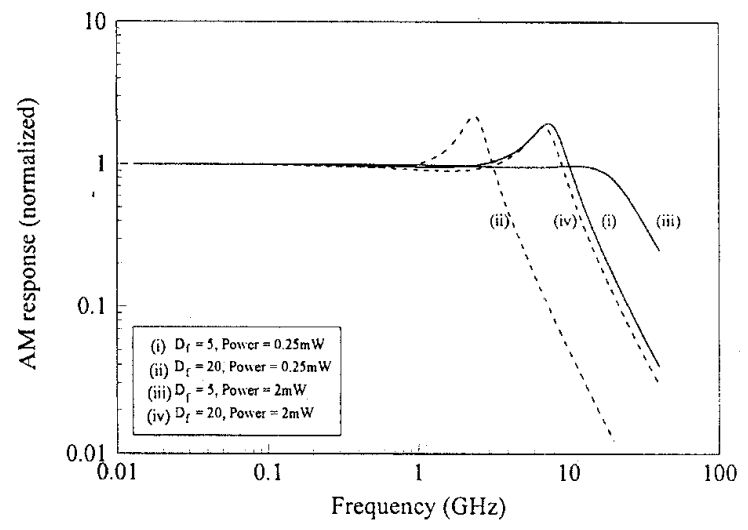

(a)

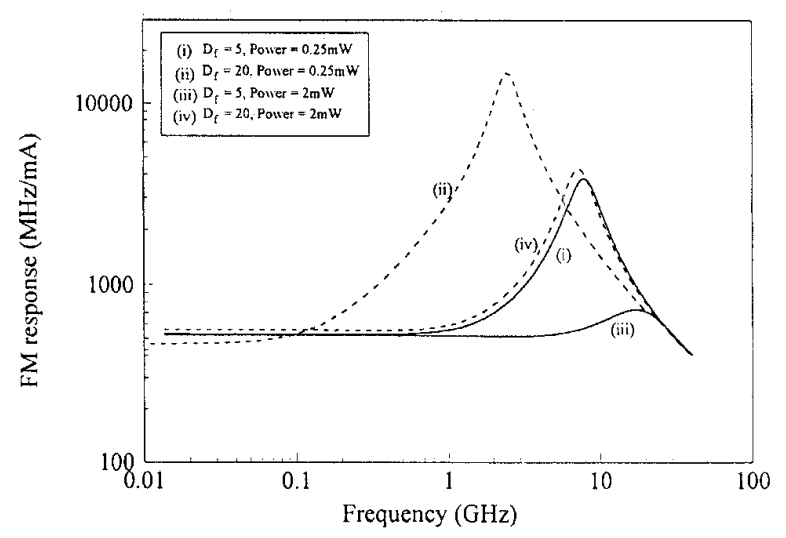

(b)

Fig. 6. The (a) AM and (b) FM responses of VCSEL's (without carrier transport) for $D_{f}=5$ and $20 \mathrm{~s}^{-1} \mathrm{~cm}^{2}$ and steady-state optical power is varied between 0.25 and $2.0 \mathrm{~mW}$.

\section{B. Influence of Carrier Transport on the Modulation Response of VCSEL's}

In this section, the influence of carrier transport on the modulation response and LSHB of VCSEL's is analyzed. First, the dynamic behavior of VCSEL's without the consideration of carrier transport is studied, then the results with carrier transport are compared with the previous analysis.

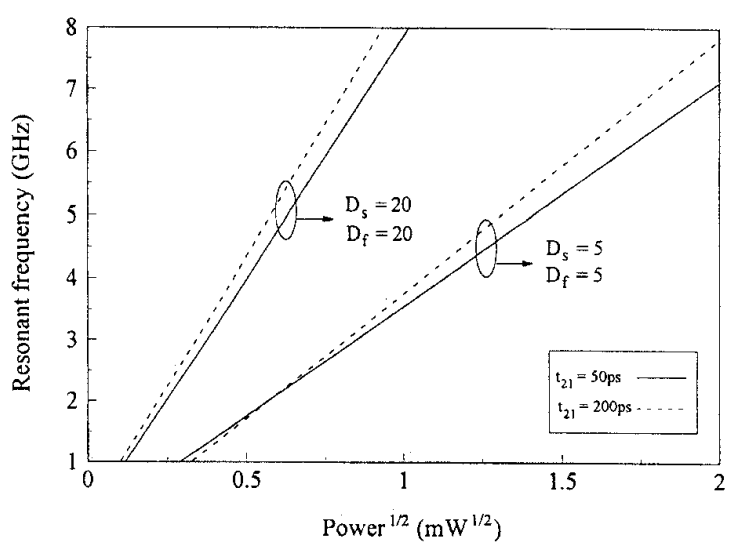

Fig. 7. Relaxation-oscillation frequency of VCSEL's under the influence of carrier transport as a function of squared root of the steady-state optical power. $D_{f}$ and $D_{s}$ are varied between 5 and $20 \mathrm{~s}^{-1} \mathrm{~cm}^{2}$. The capture time, $t_{12}$, is set to $20 \mathrm{ps}$, and the thermionic emission time $t_{21}$ is varied between 50 (solid line) and $200 \mathrm{ps}$ (dashed line)

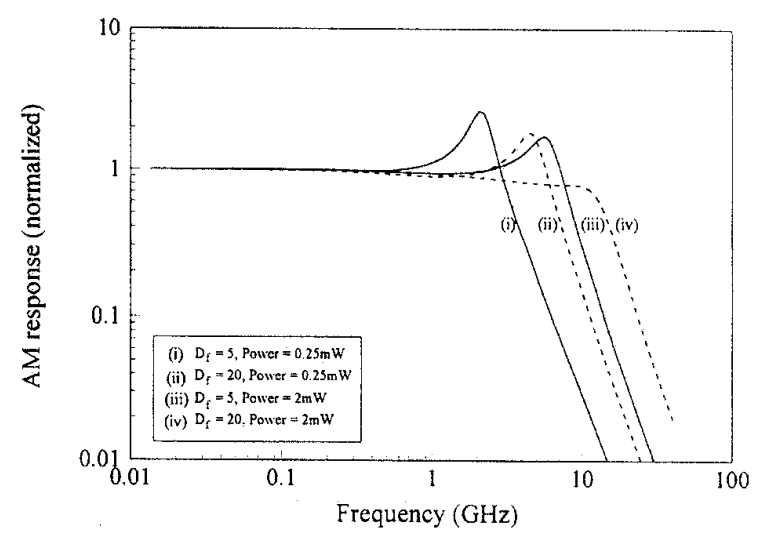

(a)

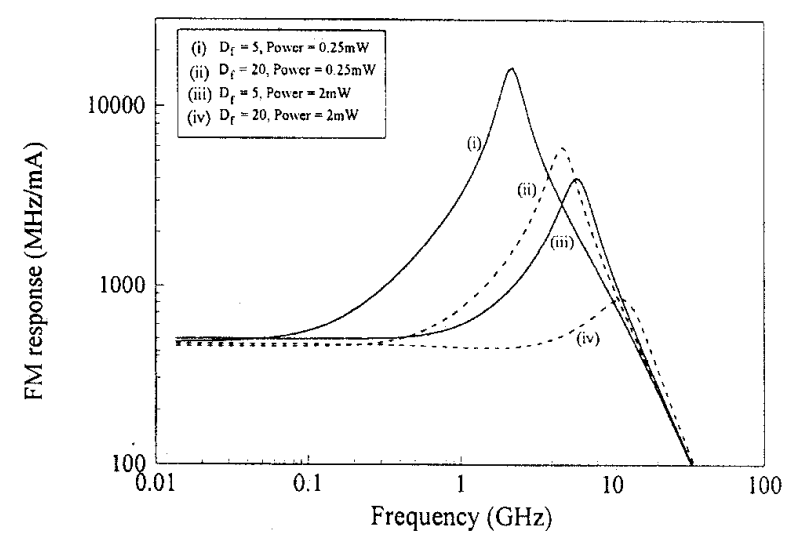

(b)

Fig. 8. Influence of carrier transport on the (a) AM and (b) FM responses of VCSEL's with $t_{12}=20 \mathrm{ps}, t_{21}=200 \mathrm{ps}$ and $D_{f}$ is varied between 5 and 20 $\mathrm{s}^{-1} \mathrm{~cm}^{2}$. The steady-state optical power is varied between 0.25 and $2.0 \mathrm{~mW}$.

Fig. 5 shows the ROF of VCSEL's for the case without the influence of carrier transport. The carrier-diffusion rate $D_{f}$ is varied between 5 and $20 \mathrm{~s}^{-1} \mathrm{~cm}^{2}$. It is noted that the ROF (at $0.25 \mathrm{~mW}$ ) is increased by more than $6 \mathrm{GHz}$ for $D_{f} \leq 15$ 


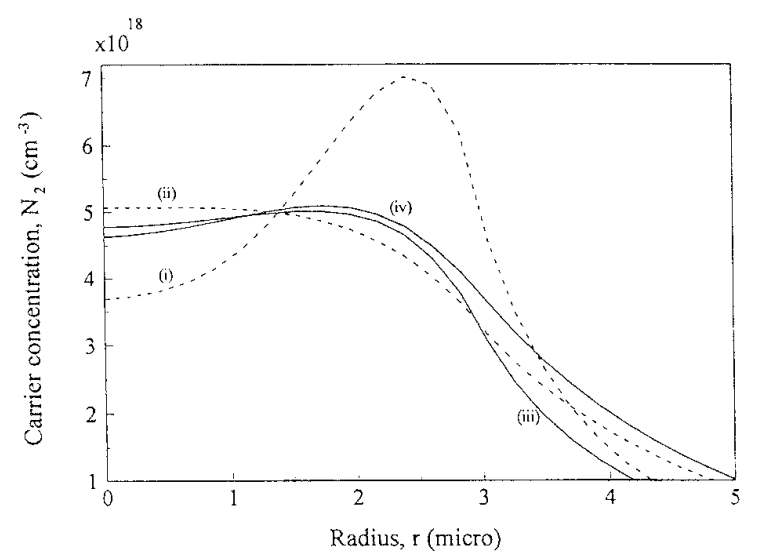

Fig. 9. Comparison of steady-state distribution of lateral carrier concentration for the cases with (solid line) and without (dotted line) carrier transport taken into calculation. (i) $D_{f}=5 \mathrm{~s}^{-1} \mathrm{~cm}^{2}$, (ii) $D_{f}=20 \mathrm{~s}^{-1} \mathrm{~cm}^{2}$, (iii) $D_{f}=D_{s}=5 \mathrm{~s}^{-1} \mathrm{~cm}^{2}$, and (iv) $D_{f}=D_{s}=20 \mathrm{~s}^{-1} \mathrm{~cm}^{2}$.

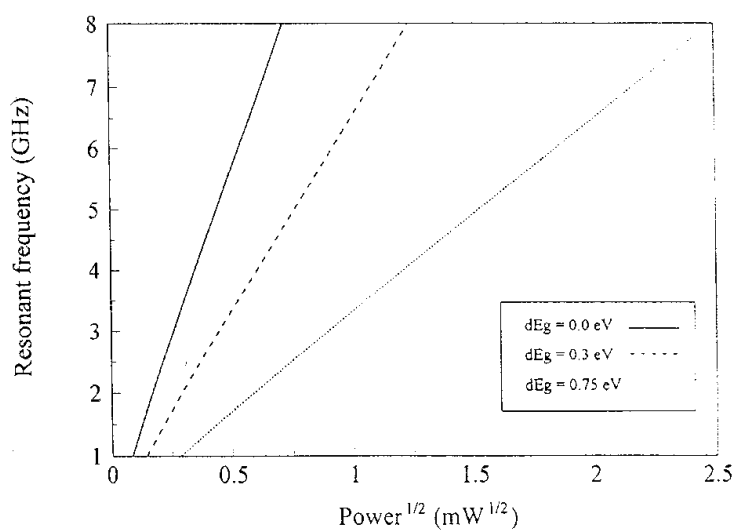

(a)

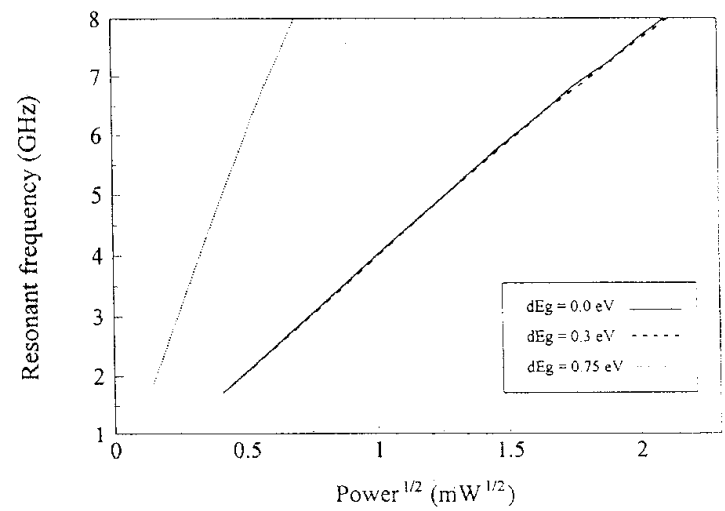

(b)

Fig. 10. Relaxation-oscillation frequency of VCSEL's under the influence of injection heating and hot carriers as a function of squared root of the steady-state optical power. In the calculations, $\Delta E_{g}$ is set to $0,0.3$, and 0.75 $\mathrm{eV}$ for the cases (a) $D_{f}=5$ and (b) $D_{f}=20 \mathrm{~s}^{-1} \mathrm{~cm}^{2}$.

$\mathrm{s}^{-1} \mathrm{~cm}^{2}$ but remains at a low value of $2 \mathrm{GHz}$ for $D_{f} \geq 15$ $\mathrm{s}^{-1} \mathrm{~cm}^{2}$. The enhancement of ROF can be explained by the severe LSHB due to the short carrier-diffusion length inside the active layer. This is because the differential gain, $a_{N} / N_{2}$,

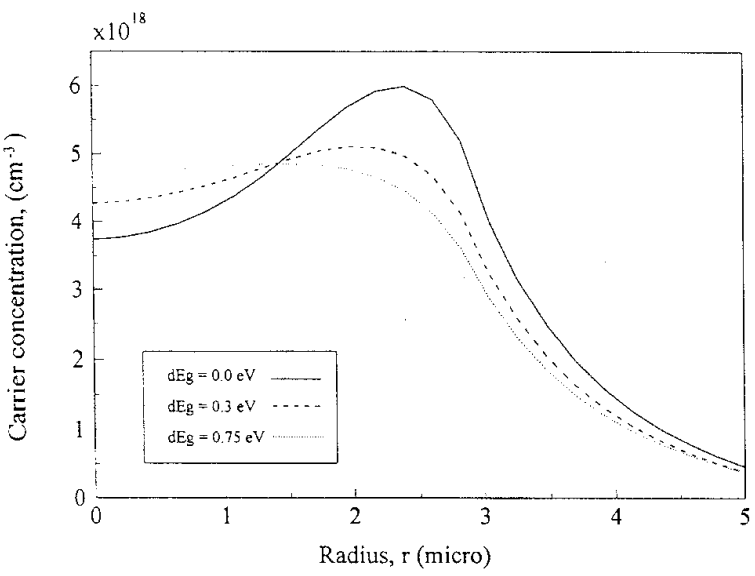

(a)

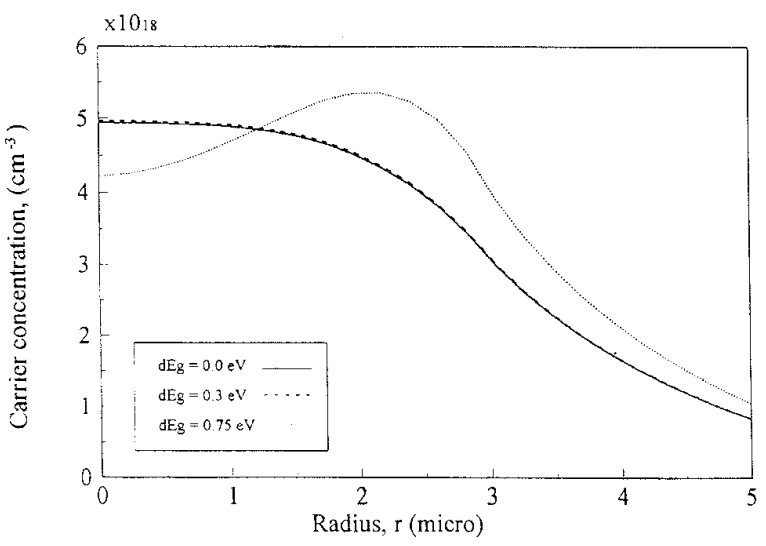

(b)

Fig. 11. Distribution of lateral carrier concentration for the cases (a) $D_{f}=$ $5 \mathrm{~s}^{-1} \mathrm{~cm}^{2}$ and (b) $D_{f}=20 \mathrm{~s}^{-1} \mathrm{~cm}^{2}$. In the calculations, $\Delta E_{g}$ is varied from 0 to $0.75 \mathrm{eV}$ and the steady-state optical power is set to $0.25 \mathrm{~mW}$.

increases with the spatial-hole burning near the center of the core and hence the ROF is enhanced. The corresponding AM and FM responses of VCSEL's are also shown in Fig. 6. The steady-state output power of the devices is maintained between 0.25 and $2.0 \mathrm{~mW}$. As we can see in Fig. $6, D_{f}$ affects the ROF and modulation bandwidth of VCSEL's, however, the carrier-diffusion rate has less influence on the rolloff of the AM response.

Fig. 7 shows the influence of carrier transport on the ROF of VCSEL's. In the calculation, the carrier diffusion and capture time, $\tau_{12}$, is set to $20 \mathrm{ps}$ and the thermionic emission time, $\tau_{21}$, is varied between 50 and 200 ps. These value of $\tau_{12}$ and $\tau_{21}$ are typical for the dimension of quantum wells and spacer layers we used in our model [4]. The carrier-diffusion coefficient of the active and spacer layers $\left(D_{f}\right.$ and $D_{s}$ are assumed to have equal magnitude) are set between 5 and 20 $\mathrm{s}^{-1} \mathrm{~cm}^{2}$. Significant influence of carrier transport on the ROF is observed when compared with Fig. 5. It is shown in Fig. 7 that the ROF (at $1 \mathrm{~mW}$ ) is increased by $5 \mathrm{GHz}$ for large carrierdiffusion rate (i.e., $D_{f}, s=20 \mathrm{~s}^{-1} \mathrm{~cm}^{2}$ ). The corresponding AM and FM responses of VCSEL's are also shown in Fig. 8 for comparison. In the calculation, the value of $\tau_{12}$ and $\tau_{21}$ 


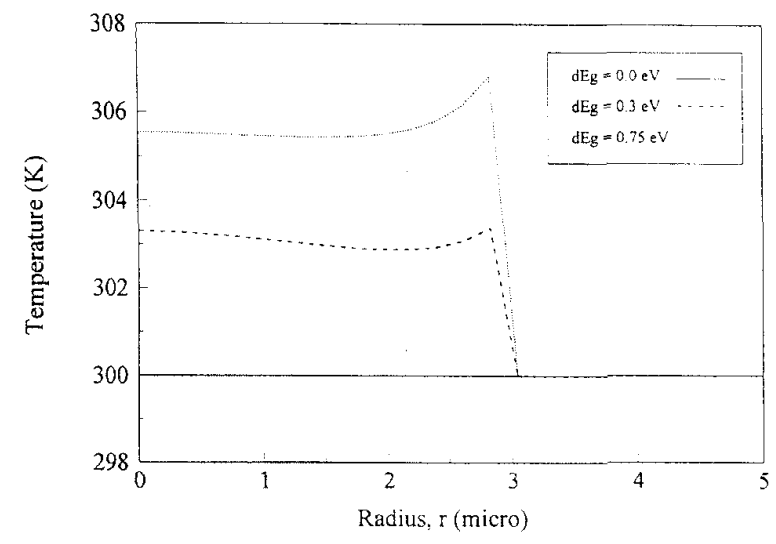

(a)

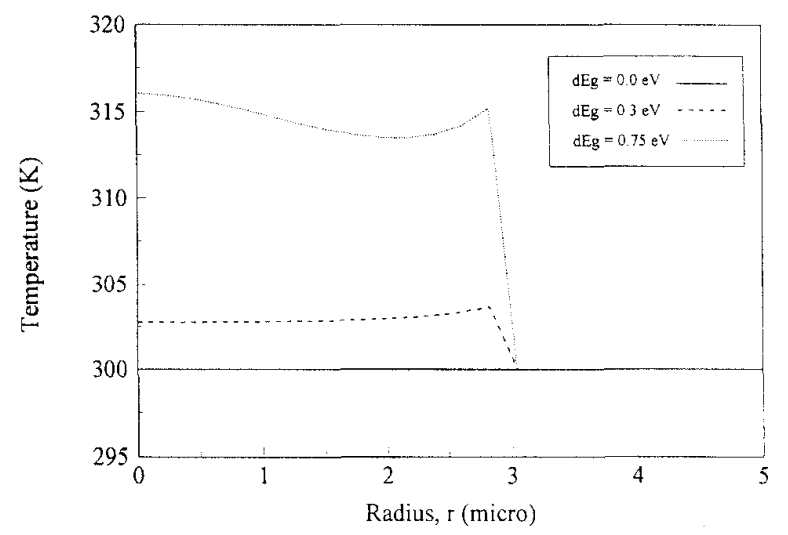

(b)

Fig. 12. Distribution of electron temperature for the cases (a) $D_{f}=5 \mathrm{~s}^{-1}$. $\mathrm{cm}^{2}$ and (b) $D_{f}=20 \mathrm{~s}^{-1} \mathrm{~cm}^{2}$. In the calculations, $\Delta E_{g}$ is varied from 0 to $0.75 \mathrm{eV}$ and the steady-state optical power is set to $0.25 \mathrm{~mW}$.

is set to $20 \mathrm{ps}$ and $200 \mathrm{ps}$, respectively. As we can see for the case $D_{f}\left(=D_{s}\right)=20 \mathrm{~s}^{-1} \mathrm{~cm}^{2}$, the ROF as well as the modulation bandwidth are enhanced by the presence of carrier transport.

Fig. 9 shows the steady-state distribution of carrier concentration for the cases with and without carrier transport taken into calculation. The output power is set to $0.25 \mathrm{~mW}$ for all cases, and the value of $\tau_{12}$ and $\tau_{21}$ is equal to $20 \mathrm{ps}$ and 200 ps, respectively. As we expected for the case without consideration of carrier transport, LSHB is enhanced for small value of $D_{f}\left(=5 \mathrm{~s}^{-1} \mathrm{~cm}^{2}\right)$ but it is minimized by the presence of transport effects. This is because carrier transport allows $N_{1}$ to refill the depletion of carrier concentration inside the active layer. Therefore, with suitable design on the dimension of spacer layers and quantum wells, LSHB can be minimized by carrier transport.

\section{Influence of Injection Heating and Hot Carriers on the Dynamic Response of VCSEL's}

In this section, we concentrate on the analysis of injection heating and hot carriers on the modulation response of VCSEL's, and the influence of carrier transport is ignored in the calculation. Fig. 10 shows the influence of injection heating



(a)

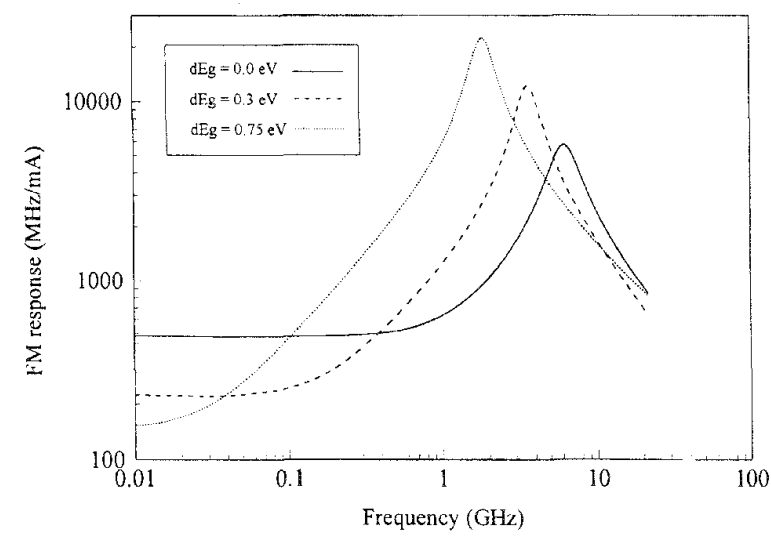

(b)

Fig. 13. Influence of injection heating and hot carriers on the (a) AM and (b) FM responses of VCSEL's with $D_{f}=5 \mathrm{~s}^{-1} \mathrm{~cm}^{2}$ and the (c) AM and (d) FM responses of VCSEL's with $D_{j}=20 \mathrm{~s}^{-1} \mathrm{~cm}^{2}$. In the calculations, $\Delta E_{g}$ is varied from 0 to $0.75 \mathrm{eV}$ and the steady-state optical power is set to $0.25 \mathrm{~mW}$.

and hot carriers on the ROF of VCSEL's. It is assumed that the lattice temperature of the quantum well is equal to $300 \mathrm{~K}$, $\Delta E_{g}$ is varied between 0 to $0.75 \mathrm{eV}$, and $D_{f}$ is set between 5 and $20 \mathrm{~s}^{-1} \mathrm{~cm}^{2} . \Delta E_{g}=0$ is the ideal case with no injection heating and hot carriers in the VCSEL's. For $\Delta E_{g}>0$ and $D_{f}=5 \mathrm{~s}^{-1} \mathrm{~cm}^{2}$, ROF (at $0.55 \mathrm{~mW}$ ) is reduced by more than $6 \mathrm{GHz}$ with the increment of $\Delta E_{g}$ [see Fig. 10(a)], but the opposite happens for $D_{f}=20 \mathrm{~s}^{-1} \mathrm{~cm}^{2}$ [see Fig. 10(b)]. As we can see, the ROF of VCSEL's is dependent strongly on both $D_{f}$ and $\Delta E_{g}$, however, in Fabry-Perot lasers the dependence on $\Delta E_{g}$ is only obvious at high output power [5].

The steady-state distribution of carrier concentrations shown in Fig. 11 for different values of $D_{f}$ and $\Delta E_{g}$. The output power of the devices is maintained at $0.25 \mathrm{~mW}$. It is shown that for $D_{f}=5 \mathrm{~s}^{-1} \mathrm{~cm}^{2}$, LSHB is reduced with the increase of $\Delta E_{g}$, however, for $D_{f}=20 \mathrm{~s}^{-1} \mathrm{~cm}^{2}$, the opposite is observed. The corresponding variation of electron temperature inside the active layer is shown in Fig. 12.

The AM and FM responses of VCSEL's under the influence of injection heating and hot carriers are also shown in Fig. 13 




(c)

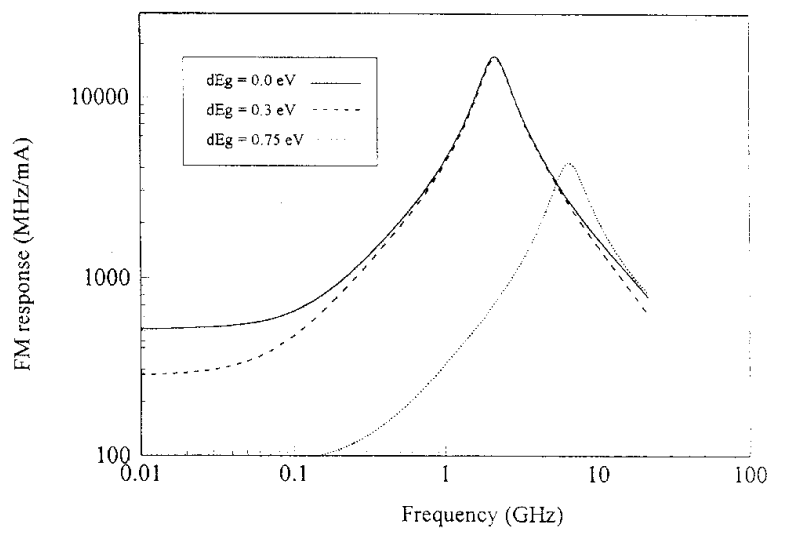

(d)

Fig. 13. (Continued.) Influence of injection heating and hot carriers on the (c) AM and (d) FM responses of VCSEL's with $D_{f}=20 \mathrm{~s}^{-1} \mathrm{~cm}^{2}$. In the calculations, $\Delta E_{g}$ is varied from 0 to $0.75 \mathrm{eV}$ and the steady-state optical power is set to $0.25 \mathrm{~mW}$.

for the cases $D_{f}=5$ and $20 \mathrm{~s}^{-1} \mathrm{~cm}^{2}$ and the values of $\Delta E_{g}$ is set to $0,0.3$, and $0.75 \mathrm{eV}$. As we can see, the magnitude of FM response is reduced significantly at low-frequency range for large values of $\Delta E_{g}(=0.75 \mathrm{eV})$. The variation of $\mathrm{ROF}$ and modulation bandwidth given in Fig. 13 is attributed to the LSHB.

\section{DISCUSSION AND CONCLUSION}

The inherent nonlinearities of VCSEL's have significant influence on its lateral carrier distribution inside the active layer. It is expected that carriers with short diffusion length enhances spatial-hole burning due to stimulate recombination. However, with the influence of carrier transport or hot carriers, a contradictory conclusion can be obtained. In the situation with carrier transport taken into consideration, LSHB is enhanced for carriers with long diffusion length. This is also true for the inclusion of injection heating and hot carriers into calculation.

We have shown LSHB enhances ROF as well as the modulation bandwidth of VCSEL's. This is because the differential gain of quantum well, $a_{N} / N_{2}$, increases with the reduction of carrier concentration. On the other hand, high-order lateral modes may be excited by LSHB at high output power. This is the tradeoff between the high modulation bandwidth and single-mode operation, but single-mode operation of VCSEL's can be maintained by using tapered optical waveguide [23] or diffused quantum-well structure [24] without reduction of LSHB.

In conclusion, a quasi-three-dimensional dynamic model of VCSEL's is developed. The detailed structure of Bragg reflectors and lateral optical confinement are considered into the model. The nonuniform distribution of optical power, carrier concentration, refractive index, as well as spontaneousemission profile are determined self-consistently. Using this model, the influence of carrier transport between the spacers and wells as well as injection heating and hot carriers are taken into calculation. It is shown that the carrier transport as well as hot carriers have significant influence on the modulation response of VCSEL's. The ROF and modulation bandwidth of VCSEL's can be altered by more that $5 \mathrm{GHz}$ by the carrier-diffusion rate and bandgap discontinuities. We have demonstrated the importance of nonlinearities on the modulation response of VCSEL's. Ignoring these effects may result in an inaccurate estimation or interpretation of the performance of VCSEL's. Therefore, this is required to consider these nonlinearities into the laser model in order to understand and to design VCSEL's for high-speed operation.

\section{REFERENCES}

[1] D. Tauber, G. Wang, R. S. Geels, J. E. Bowers, and L. A. Coldern, "Large and small signal dynamics of vertical cavity surface emitting lasers," Appl. Phys. Lett., vol. 62, pp. 325-327, 1993.

[2] G. Shtengel, H. Temkin, P. Brusenbach, T. Uchida, M. Kim, C. Parsons, W. E. Quinn, and S. E. Swirhun, "High speed vertical cavity surface emitting laser," IEEE Photon. Technol. Lett., vol. 5, pp. 1359-1362, 1993.

53] R. S. Tucker and D. J. Pope, "Circuit modeling of the effect of diffusion on damping in a narrow-stripe semiconductor lasers," IEEE J. Quantum Electron., vol. QE-19, pp. 1179-1183, 1983.

[4] R. Nagarajan, M. Ishikawa, T. Fukushima, R. S. Geels, and J. E. Bowers, "High speed quantum-well lasers and carrier transport effects," IEEE J. Quantum Electron., vol. 28, pp. 1990-2007, 1992.

[5] L. F. Lester and B. K. Ridley, "Hot carrier and frequency response of quantum well lasers," J. Appl. Phys., vol. 72, pp. 2579-2588, 1992.

[6] J. W. Scott, R. S. Geels, S. W. Corzine, and L. A. Coldren, "Modeling temperature effects and spatial hole burning to optimize vertical cavity surface emitting laser performance," IEEE J. Quantum Electron., vol. 29, pp. 1295-1308, 1993

[7] T. G. Dziura, Y. J. Yang, R. Fernandez, T. Bardin, and S. C. Wang, "High speed modulation characteristics of Helium-implanted Zincdiffused vertical cavity surface emittin lasers," IEEE Photon. Technol. Lett., vol. 5, pp. 1270-1292, 1993.

[8] F. S. Choa, Y. H. Lee, T. L. Koch, C. A. Burrus, B. Tell, J. L. Jewell, and R. E. Leibenguth, "High speed modulation of vertical cavity surface emitting lasers," IEEE Photon. Technol. Lett., vol. 3, pp. 697-699, 199 I.

[9] A. Valle, J. Sarma, and K. A. Shore, "Spatial hole burning effects on the dynamics of vertical cavity surface emitting laser diodes," IEEE $J$. Quantum Electron., vol. 31, pp. 1423-1431, 1995.

[10] I. Vurgaftman and J. Singh, "Steady-state and transient characteristics of microcavity surface emitting lasers with compressively strained quantum well active regions," IEEE J. Quantum Electron., vol. 31, pp. 852-863, 1995.

[11] W. Jiang, D. J. Derickson, and J. E. Bower, "Analysis of laser pulse chirping in mode locked vertical cavity surface emitting lasers," IEEE J. Ouantum Electron., vol. 29, pp. 1309-1318, 1993.

[12] H. Yokoyama and S. B. Brorson, "Rate eqaution analysis of microcavity lasers," J. Appl. Phys., vol. 66, pp. 4801-4805, 1989. 
[13] M. J. Adams, Introduction to Optical Waveguide. New York: Wiley, 1981.

[14] L. A. Coldren and T. L. Koch, "Analysis and design of coupled cavity lasers-Part I: Threshold gain analysis and design guidelines," IEEE $J$. Quantum Electron., vol. QE-20, pp. 659-670, 1984.

[15] A. W. Snyder and J. D. Love, Optical Waveguide Theory. London, U.K.: Chapman \& Hall, 1983, ch. 13.

[16] N. K. Dutta, "Analysis of current spreading, carrier diffusion, and transverse mode guiding in surface emitting lasers," J. Appl. Phys, vol. 68, pp. 1961-1963, 1990.

[17] L. M. Zhang, S. F. Yu, M. C. Nowell, D. D. Marcenac, J. E. Carroll, and R. G. S. Plumb, "Dynamic analysis of radiation and side-mode suppression in a second-order DFB laser using time domain large signal travelling wave model," IEEE J. Quantum Electron., vol. 30, pp. $1389-1395,1994$

[18] E. H. Li and K. S. Chan, "Laser gain and current density in a disordered AlGaAs/GaAs quantum well," Electron. Lett., vol. 29, pp. 1233-1234. 1993.

[19] D. J. Jones, L. M. Zhang, J. E. Carroll, and D. D. Marcenac, "Dynamics of monolithic passively mode locked semiconductor lasers," IEEE $J$. Quantum Electron., vol. 31, pp. 1051-1058, 1995.

[20] C. H. Henry, R. A. Logan, and A. Bertness, "Spectral dependence of the change in refractive index due to carrier injection in GaAs lasers," J. Appl. Phys., vol. 52, pp. 4457-4461, 1981.

[21] T. Baba, T. Hamano, F. Doyama, and K. Iga, "Spontaneous emission factor of a microcavity DBR surface emitting laser (II)-Effects of electron quantum confinement," IEEE J. Quantum Electron., vol. 28, pp. $1310-1319,1992$.

[22] R. S. Zory, Ed., Quantum Well Lasers. New York: Academic, 1993, ch. 1 .
[23] J. P. Zhang, "Single mode power and modal behavior in buried vertical cavity surface emitting lasers," Proc. Inst. Elect. Eng. Optoelectron., vol. 142 , no. 2,1995 , pp. 87-93.

[24] C. W. Lo, S. F. Yu, and E. H. Li, "Vertical cavity surface emitting semiconductor lasers with diffused quantum wells," presented at the IEEE TENCON'95 on Microelectronics and VLSI.

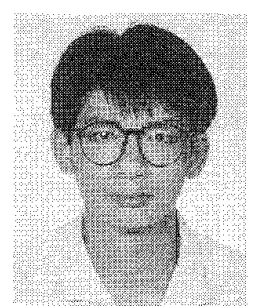

S. F. Yu received the B.Eng. degree (with Departmental Prize) in electronic engineering from London University, University College, U.K., in 1990 and the Ph.D. degree in optoelectronics from Cambridge University, Robinson College, U.K., in 1993.

In 1994, he joined the Department of Electrical \& Electronic Engineering, University of Hong Kong, where he is a Lecturer. His main research topics include wavelength selectivity of grating coupled waveguides, transient properties of semiconductorlaser diodes, and design of optoelectronic integrated circuit. He currently conducts the development of high-performance semiconductor lasers for the application in high-speed communication systems. He is also involved in the investigation of the influence of acoustic wave in semiconductor materials.

$\mathrm{He}$ is a Fellow and Honorary Scholar of Cambridge Commonwealth Trust and held a Croucher Foundation scholarship and an oversea research student award while studying for the doctoral program. 